\title{
Psychometric Evaluation of the Differentiation of Self Inventory- Revised and Mental Health Inventory-18 for Filipinos
}

\author{
Benedict G. Antazo \\ bgantazo@gmail.com
}

\begin{abstract}
The purpose of this study was to evaluate the psychometric properties and cross-cultural validity of the Differentiation of Self Inventory-Revised (Skowron \& Schmitt, 2003) and Mental Health Inventory-18 (Veit \& Ware, 1983) and to determine whether the Bowenian construct of differentiation could predict general mental health. In a sample of 322 Filipino adults, exploratory factor analyses and regression analyses were performed to determine the construct validity and strength of associations between the two concepts. Results of the EFA suggested a new three-factor model (3F-DSI-R) for the DSI-R while supporting the original two-factor model of the MHI-18. Regression analyses indicated that selfdifferentiation and its dimensions are significant predictors of mental health. Findings suggest that highly differentiated individuals tend to display better overall mental health. Lastly, implications for theory, practice, and directions for future research are discussed.
\end{abstract}

Keywords: Marital and Family Therapy, Family Systems Theory, Differentiation of Self, Mental Health, Filipino Adults

\section{Introduction}

The family systems theory of Murray Bowen $(1966,1976)$, which currently serves as the foundation of mainstream family therapy (Goldenberg \& Goldenberg, 2012) proposes eight interlocking concepts that determine how family processes are formed (Haefner, 2014) and assumes that anxiety is a result of how proximal or distant an individual is to his/her family (Brown, 1999). While some aspects of Bowen's theory have been difficult to validate due to the challenges in the development of psychometric measures, one construct - the differentiation of self - continues to make theoretical advances (Skowron \& Schmitt, 2003). Central to the theory, the differentiation of self refers to "the ability of a person to distinguish him/herself from the family of origin on a personal and intellectual level" (Bowen, 1978, in Haefner, 2014, p.1). The said concept can be separated into two levels, the intrapsychic - which refers to self-regulation; one's ability to differentiate between emotional and intellectual processes (Skowron \& Friedlander, 1998; Haefner, 2014), and the interpersonal-which refers to one's comfortability between 
autonomy and intimacy with others (Peleg-Popko, 2002; Skowron \& Schmitt, 2003). Greater differentiation leads one to take "I" positions in important relationships while considering his/her connection with others (Bowen, 1978, in Peleg-Popko. 2002; Tuason \& Friedlander, 2000; Haefner, 2014). Vancea (2013) characterized the highly differentiated self as: highly autonomous especially in dealing with significant others, possesses the capacity to distinguish between emotions and thoughts, and flexible and adaptive in dealing with one's emotions. To concretize the said construct, Skowron and Friedlander developed the Differentiation of Self Inventory in 1998. It consisted of four subscales and had a total of 43 items. This was further developed in 2003 when the Differentiation of Self Inventory-Revised was released by Skowron and Schmitt. The new subscale included new items on the Fusion subscale and consisted of 46 items.

Mental health has been defined by the World Health Organization [WHO] as "a state of well-being in which the individual realizes his or her own abilities, can cope with the normal stresses of life, can work productively and fruitfully, and is able to make a contribution to his or her community" (WHO, 2001, p. 1, in WHO, 2004). Individuals who possess good mental health are able to fulfill key functions such as learning, expressing emotions, and forming meaningful connections with others. In an attempt to develop an objective measure of mental health, Veit and Ware (1983) introduced the mental health inventory-36. A two-factor model, it consisted of the psychological distress and psychological well-being dimensions. Positive states comprised the psychological well-being scale while the negative states were classified into the psychological distress scale. On the initial findings, the psychological distress scale consisted of three subscales - anxiety, depression, and behavior control, while the psychological well-being scale consisted of two - general positive affect and emotional ties. Following the MHI's revision from 38 to 18 item inventory, the emotional ties subscale was removed from the instrument.

Several studies have demonstrated the empirical validity of the Bowenian construct of differentiation. For example, differentiation of self has been positively associated with psychological well-being (Chung \& Gale 2006; Skowron, Stanley, \& Shapiro, 2009; Sohrabi, Asadi, Habibollahzade, \& PanaAli, 2013), while an inverse relationship psychological distress has been established (Bartle-Haring, Rosen, \& Stith, 2002; Peleg-Popko, 2002; Aldea \& Rice, 2003). That is, highly differentiated individuals tend to be more satisfied with life in general and are less prone to mental health concerns. Yet despite growing evidence on the validity of differentiation, recent literature (Lam \& Chan-So, 2013; Lee \& Johnson, 2017) suggested issues with its crosscultural applicability, particularly with the fusion with others dimension. These studies have invalidated the applicability of the original four-factor model of the DSI-R when used with individuals coming from collectivistic contexts and instead proposed the use of alternative DSI-R scales with varying factor structures.

In light of the questions regarding the applicability of the Bowenian construct of differentiation, as measured by the DSI-R (Skowron \& Schmitt, 2003) across cultures, this research attempted to examine its cross-cultural validity, particularly when used in the Philippine context. Further, this study attempts to evaluate the applicability of the MHI-18 (Veit \& Ware, 1983) when used to measure the overall mental health of Filipinos. Lastly, associations between differentiation and general mental health are tested, in addition to determining whether an individual's degree of differentiation can be a significant predictor of his general mental health. 


\section{Method}

\section{Sample}

The initial sample consisted of 327 Filipinos. However, 5 responses were removed due to being invalid or incomplete thus resulting in a total of 322. Participant ages ranged from 25-66 (M $=34.80, \mathrm{SD}=9.73) .184(57.14 \%)$ of the respondents were females while 138 were males (42.86\%). As for civil status, 191 (59.32\%) were single, 127 (39.44\%) were married, 1 was separated $(.31 \%)$ and $3(.93 \%)$ were widowed. Eligibility criteria required participants to be at least 25 years old and be a natural-born Filipino who is currently living or has lived a majority of his or her life in the Philippines.

\section{Instruments}

Differentiation of Self Inventory-Revised. The original 43-item Differentiation of Self Inventory (DSI; Skowron \& Friedlander, 1998), a self-report instrument for individuals aged 25 and above, consisted of four subscales which were designed to measure the intrapsychic (thinkingfeeling) and interpersonal (separateness-togetherness) dimensions of differentiation. The intrapsychic dimension consisted of the Emotional Reactivity (ER) and "I" Position (IP) subscales while the interpersonal dimension consisted of the Emotional Cutoff (EC) and Fusion with Others (FO) subscales. According to Jankowski and Hooper (2012), the ER subscale measures one's ability to recognize and regulate his/her emotions; the IP subscale evaluates an individual's ability to maintain his/her stance, especially in times of social pressure; the EC subscale assesses one's tendency to distance him/herself from others to reduce anxiety; and the FO subscale estimates the amount of emotional involvement a person has in his/her significant relationships. Subscale scores are computed by getting the average of each item number thus range from 1 (not at all true of me) to 6 (very true of me). Total DSI-R score is the average of all subscale scores ([ER + IP + EC + FO]/4). Higher scores indicate better degree of self-differentiation. To assess the DSI's validity, Skowron and Friedlander (1998) conducted a confirmatory factor analysis (CFA) which resulted in the affirmation of their hypothesis on the four-factor model. Estimates using Cronbach Alpha indicated high internal consistency: $\mathrm{ER}=.84 ; \mathrm{IP}=.83 ; \mathrm{EC}=82 ; \mathrm{FO}=.74$; Total $\mathrm{DSI}=.88$. Further studies on the DSI supported its validity through direct association, mediation, and moderation models (see Skowron, 2000; Bohlander, 1999; Jenkins, Buboltz, Schwartz, \& Johnson, 2005; Skowron et al., 2009) while some have displayed mixed results (see Tuason \& Friedlander, 2000; Skowron, Wester, \& Azen, 2004). Despite empirical support, issues with the FO subscale prompted the development of a revised 12-tem FO scale (Jankowski \& Hooper, 2012) which resulted in the 46-item Differentiation of Self Inventory-Revised (DSI-R; Skowron \& Schmitt, 2003). The DSI-R displayed improved validity and reliability estimates: Alphas of: ER $=.89$; IP $=.81 ; \mathrm{EC}=.84 ; \mathrm{FO}=.86 ;$ Total DSI-R $=.92$. According to Lee and Johnson (2017), researches involving the DSI-R such as those of Skowron and Dendy (2004) displayed promising results. However, Existing literature still presents issues regarding the validity and internal consistency of the FO subscale (Knauth \& Skowron, 2004; Jankowski \& Hooper, 2012). 
Mental Health Inventory-18. The Mental Health Inventory-18 (MHI-18) is a shortened version of the original Mental Health Inventory-36 (MHI-36) which was developed by Veit and Ware in 1983. Intended for use in general populations, it measures the general mental health of individuals through its two dimensions, psychological distress and psychological well-being. Psychological distress dimension is composed of anxiety (ANX), depression (DEP), and behavioral control (BHC) subscales. Psychological well-being dimension is composed of the positive affect (POS) subscale. Subscale scores range from 1 (all of the time) to 6 (none of the time). These scores are then converted to total subscale scores with the equation [(MeanSubscaleScore-1)*100]/5. Total MHI score is the mean between the total scores of all subscales. Total scores will yield results between 0 (lowest) to 100 (highest). Higher scores on the ANX, DEP, and BHC subscales indicate lesser tendencies of anxiety, depression, and loss of behavioral control while higher scores on POS indicate better positive affect. Overall, higher scores indicate better general mental health. Recent research, such as Meybodi et al.'s (2011) study on a Farsi version of the MHI-18 and Yuvaraj, Poornima, \& Rashmi's (2016) research on the use of the MHI-18 as an assessment tool for overall mental health among medical students in India, support the validity, internal consistency, and cross-cultural applicability of the MHI-18.

\section{Procedure}

Data was primarily gathered through Google Forms. The link to the form was posted in various groups across different social media platforms. Clicking the link redirected potential participants to a webpage that consisted of an informed consent, a demographic questionnaire, and a digitalized version of the DSI-R and MHI-18. All fields were required to be filled up except participant name and the MHI-18 (as it provided respondents the option to skip items). In the final analysis for data inclusion, responses which were invalid (e.g. all items have a score of 1, all scales) and incomplete (more than half of MHI-18 was not answered) were removed from the final dataset. No participant withdrew from the study after the completion of the online form.

\section{Results}

\section{Exploratory Factor Analysis}

\section{Differentiation of Self Inventory-Revised}

Validity of the DSI-R was examined using principal axis factoring extraction with promax rotation. Initial factor analysis (FA) was based on eigenvalues $(>1)$ and only the items with loadings above .40 on a single factor were accepted. Initial FA which included all 46 items resulted in a twelve-factor solution. This proved to be a complicated model due multiple items without loadings above . 40 and single item factors thus was not adopted. Following the research of Knauth and Skowron (2004), the six-factor solution was attempted. All 46 items underwent a forced extraction of six factors, which also experienced the same issues as the twelve-factor solution. Due to the multiple issues of both factor models, the assessment of factors based on eigenvalue $(>1)$ was abandoned. An examination of the scree plot followed which resulted in a three-factor solution, a model consistent with recent literature (Jankowski \& Hooper, 2012; Lee \& Johnson, 
2017). All three factors accounted for $32.94 \%$ of the total variance. Several items without factor loadings above .40 on the rotated model $(1,2,5,6,10,14,17,25,27,31,33,35,37,39,40,41)$ were deleted. Items 13 (FO) and 18 (ER), which loaded on factor 2 (EI), were also deleted due to conceptual inconsistency. Similar to the findings of Lee and Johnson (2017), a number of deleted items appeared to be context free. Factor loadings for the new three-factor solution are presented in Table 1. Factors were labelled (1) Interpersonal Sensitivity, (2) Emotional Isolation, and (3) Sense of Self. Internal consistency of the whole scale and each subscale were measured, resulting in Alphas of: .81 (Interpersonal Sensitivity), .77 (Emotional Isolation), .74 (Sense of Self), .82 (Total). Factor analysis results and relatively high internal consistency suggest the viability of the 28-item, three-factor solution model as an alternative to the original DSI-R in the Philippine context.

Table 1. Factor loadings for the three-factor solution.

\begin{tabular}{|c|c|c|c|}
\hline \multirow[b]{2}{*}{ DSI-R Items } & \multicolumn{3}{|c|}{ Factor } \\
\hline & Interpersonal Sensitivity & Emotional Isolation & Sense of Self \\
\hline 1. ER Item 21 & .42 & & \\
\hline 2. ER Item 26 & .55 & & \\
\hline 3. ER Item 30 & .53 & & \\
\hline 4. ER Item 34 & .45 & & \\
\hline 5. ER Item 38 & .45 & & \\
\hline 6. FO Item 9 & .69 & & \\
\hline 7. FO Item 22 & .71 & & \\
\hline 8. FO Item 29 & .51 & & \\
\hline 9. FO Item 44 & .47 & & \\
\hline 10. FO Item 45 & .42 & & \\
\hline 11. FO Item 46 & .53 & & \\
\hline 12. EC Item 3 & & .49 & \\
\hline 13. EC Item 8 & & .54 & \\
\hline 14. EC Item 12 & & .49 & \\
\hline 15. EC Item 16 & & .57 & \\
\hline 16. EC Item 20 & & .56 & \\
\hline 17. EC Item 24 & & .49 & \\
\hline 18. EC Item 28 & & .52 & \\
\hline 19. EC Item 32 & & .43 & \\
\hline 20. EC Item 36 & & .47 & \\
\hline 21. EC Item 42 & & .51 & \\
\hline 22. IP Item 4 & & & .60 \\
\hline 23. IP Item 7 & & & .59 \\
\hline 24. IP Item 11 & & & .42 \\
\hline 25. IP Item 15 & & & .53 \\
\hline 26. IP Item 19 & & & .58 \\
\hline 27. IP Item 23 & & & .49 \\
\hline 28. IP Item 43 & & & .61 \\
\hline
\end{tabular}




\section{Mental Health Inventory-18}

Validity of the MHI-18 was examined using principal axis factoring extraction and promax rotation. Following eigenvalue $(>1)$ and scree plot examination, data analysis resulted in a twofactor solution which accounted for $60.66 \%$ of total variance. Factor loadings are shown in Table 2. Test of internal consistency resulted in alphas of: .91 (Psychological Well-Being), .91 (Psychological Distress), .93 (Total). Factor analysis and high reliability estimates suggest the applicability of the MHI-18 in the Philippine context.

Table 2. Factor loadings for the two-factor solution.

\begin{tabular}{|c|c|c|}
\hline \multirow[b]{2}{*}{$\begin{array}{l}\text { MHI-18 } \\
\text { Items }\end{array}$} & \multicolumn{2}{|c|}{ Factor } \\
\hline & $\begin{array}{l}\text { Psychological } \\
\text { Well-Being }\end{array}$ & $\begin{array}{l}\text { Psychological } \\
\text { Distress }\end{array}$ \\
\hline 1 & .72 & \\
\hline 3 & .79 & \\
\hline 5 & .63 & \\
\hline 7 & .81 & \\
\hline 8 & .77 & \\
\hline 10 & .65 & \\
\hline 13 & .84 & \\
\hline 15 & .86 & \\
\hline 2 & & .48 \\
\hline 4 & & .73 \\
\hline 6 & & .75 \\
\hline 9 & & .79 \\
\hline 11 & & .80 \\
\hline 12 & & .82 \\
\hline 14 & & .65 \\
\hline 16 & & .43 \\
\hline 17 & & .55 \\
\hline 18 & & .84 \\
\hline
\end{tabular}

\section{Test of Construct Validity and Predictability}

Correlations between the three-factor DSI-R and MHI-18 were assessed to examine validity. Further, simple and multiple regression analyses were conducted to determine whether self-differentiation can be used as a significant predictor for general mental health. Regression assumptions have been tested prior to data analysis. For the simple regression, the total scores of the 3F-DSI-R were entered as the independent variable, while the MHI-18 converted scores were entered as the dependent variable. Results indicate a significant regression equation, $(\mathrm{F}(1,320)=$ $156.27, \mathrm{p}<.001$ ), with an $\mathrm{R}^{2}$ value of .33 . For the multiple regression, the three subscales (IS, EI, $\mathrm{SS}$ ) of the three-factor DSI-R were entered as predictor variables. Analysis also found a significant regression equation, $(\mathrm{F}(3,318)=55.582 \mathrm{p}<.001)$ with an $\mathrm{R}^{2}$ value of .34 . T tests indicated that all 3 factors were significant predictors of mental health: IS $(\beta=.17, \mathrm{t}(318)=3.46, \mathrm{p}=.001)$; EI $(\beta=$ $.34, \mathrm{t}(318)=7.10, \mathrm{p}<.001) ; \mathrm{SS}(\beta=.34 . \mathrm{t}(318)=7.34, \mathrm{p}<.001)$. 


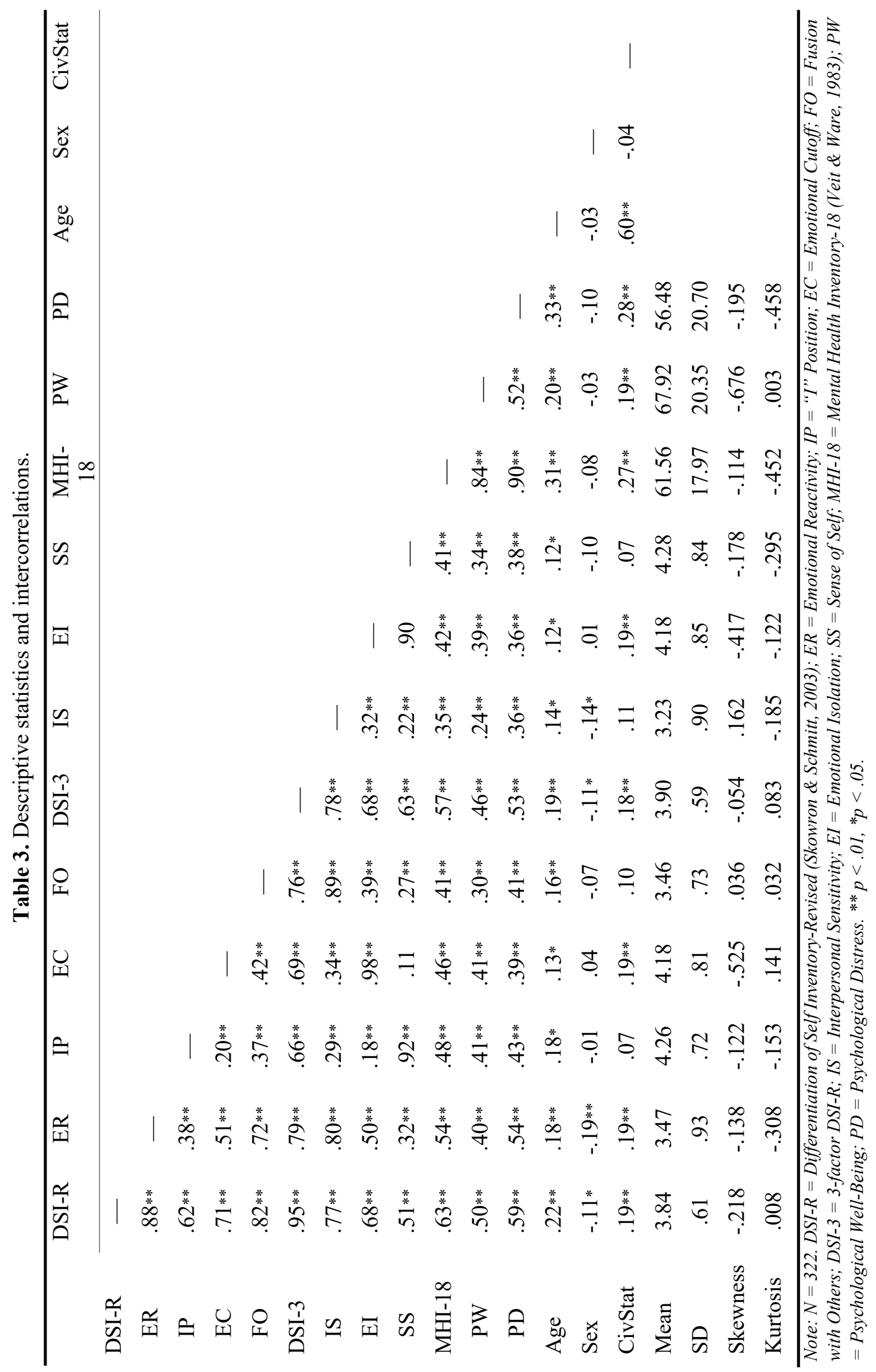




\section{Discussion}

The two primary goals of this investigation were to (1) evaluate the psychometric properties of the DSI-R (Skowron \& Schmitt, 2003) and Mental Health Inventory-18 (Veit \& Ware, 1983) when used in the Philippine context and (2) determine the relationship between the Bowenian concept of self-differentiation and general mental health. Results of both studies are discussed in the following paragraphs.

Regarding the psychometric evaluation of the DSI-R, findings suggested that the original four-factor model of the DSI-R was not applicable to Filipinos. As an alternative, the use of a three-factor model is proposed. The three-factor solution was derived through exploratory factor analysis and consists of three factors: interpersonal sensitivity, emotional isolation, and sense of self. Intercorrelations between the three-factor DSI-R (3F-DSI-R) and the original DSI-R show exceptionally high $\mathrm{R}$ values: .79 (Interpersonal Sensitivity - Emotional Reactivity), .76 (Interpersonal Sensitivity - Fusion with Others), .98 (Emotional Isolation - Emotional Cutoff), .92 (Sense of Self - "I" Position), 95 (three-factor DSI-R - four-factor DSI-R). Internal consistency estimates are also relatively high and alphas between the two scales seem to be consistent: .85 (ER), .75 (IP), .78 (EC), .73 (FO), .90 (DSI-R); .81 (IS), .77 (EI), .74 (SS), .82 (3F-DSI-R). Intercorrelations and internal consistency suggest that the three-factor DSI-R is not theoretically distant from the original DSI-R of Skowron and Schmitt (2003). However, the three-factor solution raised an issue on the validity of the FO subscale, consistent with recent literature (Jankowski \& Hooper, 2012; Lee \& Johnson, 2017). This may be due to the norm population of the DSI-R, which was conducted on individuals within an individualistic society. Compared to those who come from individualistic cultures, Filipinos - who are influenced by a collectivistic culture - may act differently when it comes to family and interpersonal relationships. Manzi, Vignoles, Regalia, and Scabini (2006, as cited in Lee \& Johnson, 2017) suggest that culture is a moderating factor on selfdifferentiation. Furthermore, according to Lee and Johnson (2017), the sentence structure of the deleted context-free items may have affected the psychometric properties of the DSI-R. This assumption is based on the suggestion of Lam and Chan-So (2013) that individuals coming from a collectivistic environment exhibit different behavior based on who they are dealing with (i.e. friends, family, significant others). The lack of distinction on social context of the deleted items may have caused difficulties with the respondents (Lee \& Johnson, 2017). Plagued with issues on validity and internal consistency of the FO subscale, the current DSI-R may not be accurate when used in the Philippine context. Instead, the 3F-DSI-R is offered as a viable alternative.

Results of the exploratory factor analysis conducted on the MHI-18 suggested a two-factor solution, supporting the original two-factor model of Veit and Ware (1983). Reliability estimates are also high: .91 (Psychological Well-Being), .91 (Psychological Distress), .93 (Total). Findings suggest that the MHI-18 (Veit \& Ware, 1983) is a valid and reliable instrument to use with Filipinos.

The present study also examined the relationship between differentiation of self and mental health using the DSI-R (Skowron \& Schmitt, 2003) and MHI-18 (Veit \& Ware, 1983). Results demonstrate a significant relationship between self-differentiation and general mental health. Furthermore, analysis indicated a direct relationship between the total 3F-DSI-R scores and psychological well-being $(\mathrm{R}=.46, \mathrm{p}<.01)$, thereby concurring with existing literature (Chung \& Gale 2006; Skowron et al., 2009; Sohrabi, et al., 2013). An inverse relationship was also 
established between one's degree of differentiation and psychological distress $(\mathrm{R}=.53, \mathrm{p}<.01$; inverse because higher scores on the psychological distress dimension of the MHI-18 indicate lower levels of anxiety, depression, and loss of behavioral control), thus supporting earlier claims on the relationship between self-differentiation and psychological distress (Bartle-Haring, Rosen, \& Stith, 2002; Peleg-Popko, 2002; Aldea \& Rice, 2003). It is also worth noting that age appears to be positively correlated to better overall mental health and lower levels of psychological distress. Lastly it was determined that self-differentiation is a significant predictor of mental health. Individuals who are highly differentiated tend to display positive affect more often and experience less anxiety, depression, and loss of behavior control.

In summary, the findings suggest the applicability both the 3F-DSI-R and the MHI-18 (Veit \& Ware, 1983) when used within a Philippine context and that an individual's degree of differentiation is a significant predictor of his or her overall mental health.

\section{Implications to the Counseling Practice}

Filipino marital and family therapists (MFTs) who utilize the family systems approach of Bowen $(1966,1976)$ may want to use the revised three-factor DSI-R when working with Filipino families for its cultural validity and appropriateness. The 3F-DSI-R is an instrument that measures an individual's overall degree of differentiation through his or her interpersonal sensitivity, emotional isolation, and sense of self. Taken together, the three factors may help mental health professionals in conceptualizing client cases. Also, the 3F-DSI can be used in conjunction with other family systems techniques (e.g. genograms) when assessing family interactions, thereby increasing the accuracy of assessment results which may then lead to better treatment outcomes.

Lastly, considering the current issues with the fusion with others construct, practitioners using Bowen's (1976) perspective must be careful in conceptualizing the role of interpersonal involvement in the Filipino differentiation process. As mentioned earlier, individuals coming from collectivistic environments might behave differently from those coming from individualistic cultures (Lee \& Johnson, 2017). Coming from a primarily collectivistic context, part of the Filipino differentiation process might be different from Bowen's original postulate on differentiation. For example, it has been said by Tuason and Friedlander (2000) that the differentiation process of individuals within collectivistic societies involve working closely with family values. Further, it is worth noting that the Filipino decision-making process involves the consideration of the welfare of others (Enriquez, 1977). Together, these practices set a question on the criterion in determining the presence of family issues (e.g. whether a family is enmeshed or not; Wong, Wong, \& Obeng, 2012, in Lee \& Johnson, 2017) among those who live within the relatively collectivistic Philippine socio-cultural context.

\section{Limitations and Future Research}

Several limitations have been determined. First, similar to the limitations of Kim et al. (2015) no objective measure of cultural values (collectivism - individualism) was used in this study. Thus, the extent of the role of cultural values in the validity of the FO subscale and in the overall differentiation process is yet to be verified. Second, the sample size included only Filipinos who are living, or have lived most of their lives, in the Philippines. Caution must be exercised 
when used with Filipinos who have already lived overseas for extended periods of time, thus subjecting them to possible acculturation. Lastly, it is worth considering the method of data collection used in this investigation might have had an influence on the results of this study. Gathered through online forms, individuals who were not familiar with the Internet were not given an opportunity to partake in the study. Moreover, the demographics questionnaire lacked questions on an individual's degree of education and socio-economic status. Due to that, possible relationships between differentiation and mental health with educational attainment and socioeconomic status were not established.

For future directions, it is suggested that researchers reevaluate the validity of the 3F-DSI$\mathrm{R}$ by conducting the same study with a larger population set, whilst including the demographic questions missed by the author. Additionally, this study focused on the psychometric properties of the DSI-R (Skowron \& Schmitt, 2003) and MHI-18 (Veit \& Ware. 1983) and the relationship between differentiation and mental health. To further strengthen the cross-cultural applicability of Bowen's (1976) construct, it is recommended to test the relationships between differentiation and other constructs in the Philippines (e.g. Differentiation and College Stress; Skowron et al., 2004). Finally, as it appears that this is the only the study that validated the psychometric properties of the DSI-R and MHI-18 for Filipinos, a cross validation using confirmatory factor analysis is highly recommended.

\section{References}

Aldea, M. A., \& Rice, K. G. (2006). The role of emotional dysregulation in perfectionism and psychological distress. Journal of Counseling Psychology, 53(4), 498.

Bartle-Haring, S., Rosen, K. H., \& Stith, S. M. (2002). Emotional reactivity and psychological distress. Journal of Adolescent Research, 17(6), 568-585.

Bohlander, R. W. (1999). Differentiation of self, need fulfillment, and psychological well-being in married men. Psychological Reports, 84(3_suppl), 1274-1280.

Bowen, M. (1966). The use of family theory in clinical practice. Comprehensive psychiatry, 7(5), 345-374.

Bowen, M. (1976). Theory in the practice of psychotherapy. In P. J. Guerin (Ed.): Family therapy: theory and practice. New York: Gardner Press.

Brown, J. (1999). Bowen family systems theory and practice: Illustration and critique. Australian and New Zealand Journal of Family Therapy, 20(2), 94-103.

Chung, H., \& Gale, J. (2006). Comparing self-differentiation and psychological well-being between Korean and European American students. Contemporary Family Therapy, 28(3), 367-381.

Enriquez, V. (1977). Filipino psychology in the Third World. Philippine Journal of Psychology, $10(1), 3-18$.

Goldenberg, H., \& Goldenberg, I. (2012). Family therapy: An overview. Cengage learning.

Haefner, J. (2014). An application of Bowen family systems theory. Issues in Mental Health Nursing, 35(11), 835-841.

Jankowski, P. J., \& Hooper, L. M. (2012). Differentiation of self: A validation study of the Bowen theory construct. Couple and Family Psychology: Research and Practice, 1(3), 226. 
Jenkins, S. M., Buboltz, W. C., Schwartz, J. P., \& Johnson, P. (2005). Differentiation of self and psychosocial development. Contemporary Family Therapy, 27(2), 251-261.

Kim, H., Prouty, A. M., Smith, D. B., Ko, M. J., Wetchler, J. L., \& Oh, J. E. (2015). Differentiation and healthy family functioning of Koreans in South Korea, South Koreans in the United States, and white Americans. Journal of marital and family therapy, 41(1), 72-85.

Knauth, D. G., \& Skowron, E. A. (2004). Psychometric evaluation of the differentiation of self inventory for adolescents. Nursing Research, 53(3), 163-171.

Lam, C. M., \& Chan-So, P. C. (2015). Validation of the Chinese version of Differentiation of Self Inventory (C-DSI). Journal of marital and family therapy, 41(1), 86-101.

Lee, H. H., \& Johnson, R. W. (2017). Assessing the Psychometric Properties of the Differentiation of Self Inventory-Revised for Asian-American Bicultural Adults. The American Journal of Family Therapy, 45(1), 51-65.

Meybodi, F. A., Saeedi, Z., Behjati, Z., Noorbala, F., Dastbaravardec, A., \& Enjedany, E. (2011). Reliability and validity of a Farsi version of 18-item Mental Health Inventory. ProcediaSocial and Behavioral Sciences, 30, 1425-1429.

Peleg-Popko, O. (2002). Bowen theory: A study of differentiation of self, social anxiety, and physiological symptoms. Contemporary Family Therapy, 24(2), 355-369.

Skowron, E. A., \& Friedlander, M. L. (1998). The Differentiation of Self Inventory: Development and initial validation. Journal of counseling psychology, 45(3), 235.

Skowron, E. A., \& Schmitt, T. A. (2003). Assessing interpersonal fusion: Reliability and validity of a new DSI fusion with others subscale. Journal of marital and family therapy, 29(2), 209-222.

Skowron, E. A., Stanley, K. L., \& Shapiro, M. D. (2009). A longitudinal perspective on differentiation of self, interpersonal and psychological well-being in young adulthood. Contemporary Family Therapy, 31(1), 3-18.

Skowron, E. A., Wester, S. R., \& Azen, R. (2004). Differentiation of self mediates college stress and adjustment. Journal of Counseling \& Development, 82(1), 69-78.

Sohrabi, R., Asadi, M., Habibollahzade, H., \& PanaAli, A. (2013). Relationship between self-differentiation in bowen's family therapy and psychological health. ProcediaSocial and Behavioral Sciences, 84, 1773-1775.

Tuason, M. T., \& Friedlander, M. L. (2000). Do parents' differentiation levels predict those of their adult children? and other tests of Bowen theory in a Philippine sample. Journal of counseling psychology, 47(1), 27.

Vancea, F. (2013). The Increase of the Differentiation Level of the Self through Unifying Personal Development. Procedia-Social and Behavioral Sciences, 78, 180-184.

Veit, C. T., \& Ware, J. E. (1983). The structure of psychological distress and well-being in general populations. Journal of Consulting and Clinical Psychology, 51(5), 730-742.

World Health Organization. (2004). Promoting mental health: Concepts, emerging evidence, practice: Summary report.

Yuvaraj, B. Y., Poornima, S., \& Rashmi, S. (2016). Screening for overall mental health status using mental health inventory amongst medical students of a government medical college in North Karnataka, India. International Journal of Community Medicine and Public Health, 3(12), 3308-3312. 